\title{
Exploiting autoimmunity unleashed by an off-label low-dose immune checkpoint blockade to treat advanced cancer
}

Tibor Bakacsa*, M.D., Ph.D., D.Sc., Ralph W Moss ${ }^{b}$, Ph.D., A. Marcell Szasz ${ }^{c}$, M.D., Ph.D., Colin C Andersond, Ph.D.

aPRET Therapeutics Ltd., 1124 Budapest, Hungary; Phone: +36 30 7265122; tiborbakacs@gmail.com

${ }^{\mathrm{b} C a n c e r}$ Communications, Inc., PO Box 1076 Lemont, PA 16851 USA; Phone: 814-238-4064; ralph@cancerdecisions.com;

'Cancer Center, Semmelweis University, 1083 Budapest, Hungary; Phone: +36 20825 9684; szasz.attila marcell@med.semmelweis-univ.hu;

${ }^{\mathrm{d} D e p a r t m e n t s}$ of Surgery and Medical Microbiology \& Immunology, Alberta Diabetes Institute, Alberta Transplant Institute, University of Alberta; Phone: 780-492-6036;

colinand@ualberta.ca

*Corresponding author: Tibor Bakacs, tiborbakacs@gmail.com;

To the memory of Melvin Cohn, founding fellow and professor emeritus of the Salk Institute for Biological Studies

\section{ABSTRACT}

As a result of the cancer immunotherapy revolution hundreds of clinical trials of the newly approved immunotherapies are now under way to improve responses. Not unexpectedly, the 2018 Nobel Prize in Physiology or Medicine was awarded to James P. Allison and Tasuku Honjo for their development of cancer therapy by blockade of co-inhibitory signals. While success stories of terminal cancer patients achieving complete remissions are accumulating, not enough research has been done into the risks of the new therapies. Since the use of immunotherapy is becoming more common, and is expected to develop into first- and second-line treatments, immunotoxicity and autoimmunity are emerging as the nemesis of immunotherapy. Immune-related adverse events (IrAEs) could affect any tissue, their incidence may reach up to $90 \%$ of patients and toxicity is dose-dependent. While the combination of two immune checkpoint inhibitors (ICls) increased efficacy, the incidence of severe adverse events was also increased. Apparently, ICls cannot be restricted to the targeted anti-tumor T cell population. The long lasting objective of cancer regression can only be achieved by paying a price: tolerance to healthy self tissues is compromised. In the face of an ipilimumab induced pan-lymphocytic activation, a therapeutic paradigm shift is required. The task is not desperately trying to put the genie back in the bottle by immune suppressive treatments, but instead harnessing the autoimmune forces by an off label lowdose combined anti-CTLA-4 and anti-PD1 antibody blockade, which is supplemented with conventional interleukin-2 stimulation and hyperthermia. The proof-of-principle of the lowdose-combination therapy was demonstrated in a heavily pre-treated triple negative breast cancer (TNBC) patient with far advanced pulmonary metastases and severe shortness of breath, who had exhausted all conventional treatment. Her pulmonary metastases went into complete remission with transient WHO I-II diarrhea and skin rash. She lived for 27 months after starting the low-dose-combination therapy. She had recurrence as a sternal mass and pleural metastases up to $3 \mathrm{~cm}$. Since the low-dose-combination protocol consists only of approved drugs and treatments, this exceptional response should instigate further research efforts. (words: 334) 
Key words: immune checkpoint inhibitors; immune-related adverse events (irAEs); tolerance; ipilimumab; nivolumab; interleukin-2; hyperthermia; low-dose-combination therapy;

\section{Highlights}

- Checkpoint inhibitors achieved regression of cancer in a minority of patients

- However, the majority suffered immune-related adverse events

- Cancer regression can only be achieved by tolerance breakdown

- Autoimmunity is emerging as the nemesis of immunotherapy

- Therefore, a therapeutic paradigm shift is required

- Autoimmune T cells can be harnessed by a low-dose combined checkpoint blockade

\section{Introduction}

The immune system is continuously in a state of delicate balance between tolerating normal tissues ("self") and attacking foreign substances. If this balance is perturbed, autoimmune reactions occur. To avoid collateral damage of normal tissues [1], natural inhibitory feedback loops, generated by co-inhibitory signals, and also referred to as immune checkpoints, reduce inflammation following immune activation [2].

For a century it was believed that the immune response can destroy anything containing a foreign substance, such as pathogens or altered host cells (e.g. cancer). Following the success of vaccines against xenogeneic infectious diseases, the tacit assumption was that host immunity would also be protective against isogeneic cancer [3]. Cancer immunotherapy trials, however, conducted with the best available science resulted in anecdotal responses such that the field of cancer immunotherapy did not quite succeed in fulfilling the great hopes of conquering cancer and began to lose credibility [4].

Studies initiated by J. P. Allison led to a clinical breakthrough via the translation of immune checkpoint inhibitors (ICls) [4] [5]. A recent special issue of Science entitled, The Cancer Immunotherapy Revolution, reviewed the newly approved immunotherapies that can manipulate components of the immune system to attack tumors [6]. Hundreds of clinical trials are under way to improve responses and success stories of terminal cancer patients defying the odds and achieving complete remissions are accumulating.

Unfortunatelly, the manipulation of the immune system has also resulted in a major safety issue: the iatrogen immune-related adverse events (IrAEs). Even the New York Times 
reported that the immune system unleashed by checkpoint blockades can attack healthy, vital organs. ${ }^{1}$ According to Prof William Murphy (University of California, Davis) not enough research has been done into the risks of the new therapies, which is a massively understudied area. The critical NYT article was quoted by the NEJM Journal Watch. ${ }^{2}$

\section{Blocking negative feedback signals (from co-inhibitors) for tumor immunotherapy was proposed in 1971}

While popular in the clinical lexicon, in terms of scientific understanding the term immune checkpoint appears to confuse more than it illuminates. It is a vague term, referring broadly to many inhibitory or stimulatory signaling pathways in lymphocytes. The immune checkpoints that have been successfully targeted in cancer have all been well defined coinhibitory receptors or their ligands. It is also surprising that the current leaders of coinhibitory blockade (the mechanism of the new immunotherapy) appear universally unaware of the scientific origin of the concept.

The discovery of co-inhibition is typically not mentioned (e.g. [7]) or has erronously been described as originating in the early 1990s with inhibitory receptors in NK cells [8] or with identification of CTLA-4 function in T cells [2] [9]. However, the first co-inhibitory receptor that was discovered and characterized was instead FcyRIIb in B cells [10] [11] [12] [13]. This receptor is not targeted in current checkblockade approaches, as promoting B cell responses is thought to be rarely effective in cancer; nevertheless, the concept of co-inhibitory checkpoints originated with these studies and theories about regulation of $\mathrm{B}$ cell responses. A currently popular concept, proposed 22 years ago [14], is that peripheral tolerance or immunity is determined by the balance of mutliple co-stimulatory vs. co-inhibitory signals, or by their temporally distinct expression pattern. The "co" in both co-stimulation and coinhibition is the key to the concept that these receptors do not act on their own, but instead only when co-engaged with activating receptors such as the TCR and BCR. This limits activation or inhibition to cells that have recently engaged or are currently engaging antigen (i.e. cells that have recently received an antigen receptor signal). This concept was an

\footnotetext{
${ }^{1}$ https://www.nytimes.com/2016/12/03/health/immunotherapycancer.html?rref=collection\%2Fsectioncollection\%2Fhealth\&action=click\&contentCollection=health\&region=ra nk\&module=package\&version=highlights\&contentPlacement=1\&pgtype=sectionfront 2 http://www.jwatch.org/fw112314/2016/12/05/cancer-immunotherapies-may-send-immune-systemoverdrive?query $=$ etoc jwonchem\&jwd=000020025654\&jspc $=\mathrm{Al}$
} 
extension of earlier proposals that both $\mathrm{T}$ and $\mathrm{B}$ cell responses are controlled by feedback inhibitory signals [15] [16] [17].

In a 1971 paper remarkably ahead of its time, Sinclair and Chan, based on their data, predicted not only that B cells have IgG Fc receptors that are inhibitory when co-engaged with $B C R$, they also predicted that blocking such negative feedback signals (from coinhibitors) would be a beneficial approach in tumor immunotherapy [10]. The concept that peripheral tolerance arises from co-inhibitory signals rather than signals from the antigen receptor alone ('signal 1') was developed by Sinclair based on his unconventional view that the antigen receptor (TCR, BCR) signal alone is activating rather than tolerogenic. Tolerance arising from signal 1 versus co-inhibition has been debated extensively at the conceptual level [16] [18] [19] [20]. The two main competing viewpoints being, 1. the proposal that coinhibitors are key to tolerance during chronic antigen exposure [14] [15] [19] and 2. the view that the raison d'être of co-inhibitors is not about tolerance but instead about the control of the magnitude and class of immune response [18] [20]. There is now extensive data supporting a role for co-inhibition in tolerance during chronic antigen exposure (a state frequently referred to as 'exhaustion') [8] [21]; however, experimentally it remains unclear whether all cases of peripheral tolerance involve a co-inhibitory signal or if signal 1 alone can be tolerogenic.

\section{Cytotoxic T lymphocyte antigen 4 (CTLA-4), was the first co-inhibitor shown to be relevant} to cancer therapy

Notwithstanding, while the ICls interrupted T cell pathways responsible for immune downregulation and mediated regression of established malignant tumors in a minority of patients, the majority suffered immune-related adverse events (irAEs).

In fact, the seminal phase III trial of Hodi et al. [22], which reported improved survival with ipilimumab in patients with metastatic melanoma with a response rate of $10.9 \%$ in 676 patients administered ipilimumab, obscured the findings that the complete response rate was only $0.2 \%$ with one patient out of 403 who received ipilimumab plus a peptide vaccine. Meanwhile, practically all the patients suffered toxicity. With the benefit of hindsight, this paper predicted correctly that tumor eradication will be associated with tolerance breakdown. "Abrogation of the function of CTLA-4 would permit CD28 to function 
unopposed and might swing the balance in favor of immune stimulation, tolerance breakdown and tumor eradication..."

Bakacs et al. critically re-considered the very same published evidence and suggested an alternative interpretation of the widespread irAEs by comparing the outcomes of the ipilimumab trials and the phase I clinical trial of a humanized 'superagonist' anti-CD28 mAb (TGN1412), which ended up in a catastrophe [23]. It was proposed that the underlying basic mechanism of actions of agonistic (anti-CD28) and inhibitory (anti-CTLA-4) immune modulatory therapies are similar such that they cannot be restricted to the targeted T cell population [24]. Anti-CTLA-4 antibodies are able to block the CTLA-4 receptors not only on tumor specific T cells but also on all activated T cells.

\section{Anti-CTLA-4 antibody blockade induces an uncontrolled T-cell activation}

We would suggest that the widespread, dose-dependent irAEs of ipilimumab can best be explained by the view that all T cells possess self reactivity [25] [24] [26] [27] [28]. The 'tonic' signal one (TCR signal) generated by positively selecting self-peptide/MHC promotes activation and homeostatic survival of T cells in the periphery. Furthermore, there is evidence for control of such tonic TCR signals by co-inhibitors [29]. Tonic signals are a form of chronic signaling that may tune T cells, establishing the threshold for the level of signals needed for activation broadly across the T cell repertoire, as postulated by Grossman and Paul [30] [31]. This is consistent with a critical role for co-inhibitors early in life to establish tolerance in the first T cells that seed the periphery [29] [32] [33]. In addition, the ability of TCRs to interact with tonic self-peptide/MHC ligands opens the possibility that co-inhibitor blockade causes T cell effector activity to spill over onto nearby healthy cells, or tumor cells that have downregulated tumor antigens. Increased collateral damage is indeed seen during immune responses where a co-inhibitor is lacking [1]. Altogether, the above concepts suggest that all T cells are temporarily activated, expressing co-inhibitors such as CTLA-4 that can then be targeted by anti-CTLA- 4 antibodies. This is consistent with aspects of the immunological homunculus concept of Irun Cohen, who suggested that the immune system continuously responds to self [34] [35] [36] [37]. In this way, the anti-CTLA-4 mab blockade induces an uncontrolled T-cell activation. It was predicted that the long lasting objective of cancer regression will be achieved only by paying a price: tolerance to healthy self tissues will be compromised. 
This prediction has been confirmed in many thousands of patients. By now Science has also acknowledged that these patients are "human experiments" of the autoimmune process [38]. Notwithstanding, we could not find a paper (other than our own) that deduced the widespread irAEs based on the similar outcomes of the TGN1412 and ipilimumab trials despite the fact that the number of ipilimumab papers has increased from 144 (in 2011) to 2585 (PubMed search as of October, 2018).

\section{In the face of widespread autoimmune toxicities, insisting that the CTLA-4 blockade is tumor specific is ignoring the obvious}

As a result of the impaired self-tolerance, irAEs may present with a broad clinical spectrum that mainly involves the gut, skin, endocrine glands, liver, and lung but can potentially affect any tissue, and their incidence may reach up to $90 \%$ of patients [39] [40]. A recent educating example for this problem is the paper of Tawbi et al., who demonstrated that nivolumab combined with ipilimumab had clinically meaningful intracranial efficacy in patients with melanoma who had untreated brain metastases [41]. However, treatment-related adverse events were reported in $97 \%$ of patients, while grade 3 or 4 adverse events occurred in $55 \%$ of patients, including events involving the central nervous system in $7 \%$, and one patient died from immune-related myocarditis. Not unexpectedly, the Nobel committee emphasized that a crucial aspect in the future development of checkpoint inhibitor therapies is to improve understanding of events leading to adverse events. ${ }^{3}$ As described by Puzanov, et al meta-analysis indicates an overall incidence of $<75 \%$ with anti-CTLA-4 monotherapy (ipilimumab), and $\leq 30 \%$ in phase 3 trials of anti-PD-1/PD-L1 agents. IrAEs of $\geq$ grade 3 severity occur in up to $43 \%$ of patients given ipilimumab and $\leq 20 \%$ given blockers of PD1/PD-L1. The incidence of irAEs with ipilimumab and pembrolizumab is dose-dependent, with greater toxicity at higher dose levels; toxicity also varies between the adjuvant and metastatic disease settings. Death due to irAEs occurred in up to $2 \%$ of patients (see in [39]). The distinct mechanisms of action of anti-CTLA-4 and anti-PD-1/anti-PD-L1 antibodies have led to trials examining combination therapies in a variety of malignancies. Unfortunately, with increased efficacy the incidence of severe adverse events also inreased. Combination of ipilimumab with nivolumab is associated with a high rate (55\%) of grade $3 / 4$ adverse events leading to discontinuation in a third of those treated. Symptoms from ICls may present as

\footnotetext{
${ }^{3}$ http://www.nobelprizemedicine.org/
} 
serious and life-threatening events, which require timely patient management and adequate therapeutic decisions [42-47]. The Oncology Nursing Society has an immunotherapy wallet card available for patients and providers, which warns that patients have a life-time risk of irAEs [40].

It is therefore very concerning that several centers experienced difficulties in patient compliance with reporting adverse events. Patients often deny their symptoms when they fear their treatment will be stopped due to irAEs [48].

In the face of widespread autoimmune toxicities, insisting that the CTLA-4 blockade is tumor specific is ignoring the obvious [49]. Clinical remission (partial or complete), or at least cancer stabilization, was noted for $60 \%$ of patients who experienced an irAE. Furthermore, a strong correlation was observed between the induction of tumor regression and grade 3/4 autoimmune toxicity corroborating the idea of coupling autoimmunity and tumor immunity [50].

Consistent with this, a new study revealed an association of irAEs with the efficacy of PD-1 inhibitors in non-small-cell lung cancer (NSCLC) with landmark analysis (which minimized leadtime bias potentially associatedwith time-dependent factors such as irAEs) and multivariable analysis [51]. This finding can be interpreted to mean that the anti-PD-1 antibodies modulate not only the activity of trained killer T lymphocytes that have migrated into tumors [2], but had also a systemic effect on T-lymphocyte immunity.

\section{latrogenic CTLA-4 blockade turns physiologic autoimmunity into a pan-lymphocytic activation}

It was originally assumed that most CTLA-4 expressing T-cells are either anti-tumor effector cells or regulatory T-cells inhibiting anti-tumor response [49]. Consequently, it was believed that CTLA-4 blockade selectively targets T-cells relevant to the anti-tumor immune response. Unfortunately, this assumption cannot be reconciled with the widespread irAEs observed in the vast majority of patients. In support of the viewpoint that CTLA-4 blockade is tumor specific, proponents cite the lack of autoimmunity in mice treated with antagonists of CTLA4 [49], arguing that releasing the brakes when almost all the T cells are 'in park' cannot promote autoimmunity. This fundamentally misunderstands differences in the activation status of the immune system between mice and humans, humans having a much more broadly activated state (many fewer cells in park) than the 'clean' mice used in laboratory 
studies [52]. The human experiments being performed with ICls have proven this point quite clearly.

Since immune cells require regular stimulation for survival [53], Bakacs et al. proposed that self-antigens, from time to time, activate T cells through an internal dialogue via a one-signal mechanism [54]. Temporarily activated T cells express CTLA-4, which is blocked by anti-CTLA4 antibodies not only on tumor specific T cells but also on all activated T cells [26]. As predicted by Hodi et al. [22], abrogation of the function of CTLA-4 results in immune stimulation, tolerance breakdown, and eventually tumor eradication.

Bakacs et al. argued, therefore, for a profound theoretical point against the consensus of experts. Since the anti-CTLA-4 immune checkpoint blockade cannot be restricted to the targeted tumor-specific $\mathrm{T}$ cell population and they induce an uncontrolled pan $\mathrm{T}$ cell activation, tolerance to healthy self tissues will be compromised. They therefore hypothesized that the anti-CTLA-4 therapy may have mechanisms similar to that occurring in inherited human CTLA4 haplo-insufficiency [28].

This theoretical proposition is consistent with the finding that inherited human CTLA4 haploinsufficiency plays a critical quantitative role for CTLA-4 in governing T and B lymphocyte homeostasis [55]. This proposition has since been validated by two new genetic diseases of CTLA-4 checkpoint insufficiency. In 2014, heterozygous, deleterious mutations in the CTLA4 gene were discovered to be the cause of a dominantly inherited immune dysregulation disorder, characterized by lymphocytic infiltration of multiple nonlymphoid organs and termed 'CTLA4 Haploinsufficiency with Autoimmune Infiltration' (CHAI). The disorder caused by deleterious biallelic mutations in LRBA has been termed LATAIE, 'LRBA deficiency with Autoantibodies, Treg defects, Autoimmune Infiltration, and Enteropathy', which emphasizes the predominant disease features. Overall, the disease resembles CHAI but presents more often with an earlier age of onset and a significantly greater disease penetrance [56] [57].

\section{Clinical phenotypes of CTLA4 gene insufficiency are eerily similar to that of the CTLA-4 blockade}

The severe clinical phenotypes of CHAI and LATAIE patients underscore the importance of the negative regulatory molecules in preventing autoimmunity, lymphoproliferation, and unnecessary tissue damage in humans. Patients with CHAI and LATAIE present with 
autoantibody-mediated cytopenias, lymphadenopathy/ splenomegaly, hypogammaglobulinemia, organ-specific autoimmunity, and lymphocytic infiltration of nonlymphoid organs. Despite varying clinical manifestations, most CHAI and LATAIE patients have lymphocytic overactivation and infiltration of at least 1 non-lymphoid organ, usually the intestine, lungs, or brain. Intestinal involvement that causes enteropathy is most common for both diseases. The lungs are the second most frequently infiltrated organ, and infiltrates in the brain are less common (see Figure 1 in [57]).

It is educating to compare the symptoms of the CTLA4 gene insufficiency to that of the iatrogenic CTLA-4 blockade (Figure 1). Screening 752 patient files among 19 skin cancer centers, Voskens et al. summarized rare and difficult-to-treat ipilimumab-induced side effects [58]. As CTLA-4 is inducible on virtually all T cells, ipilimumab-induced irAEs can virtually affect any organ system or tissues. A total of 88 rare AEs were observed in 82 patients, affecting the skin, endocrine system, nervous system, liver, respiratory tract, gastrointestinal tract, pancreas, sinuses, renal system, musculoskeletal system, heart, eyes, and upper extremities. In addition, a systemic grade IV anaphylactic reaction and a fatal case of tumor mass liquefaction were also reported [58].

Figure 1. Immune-related adverse events (irAEs) of checkpoint inhibitors and clinical phenotypes of CTLA4 gene insufficiency are very similar.

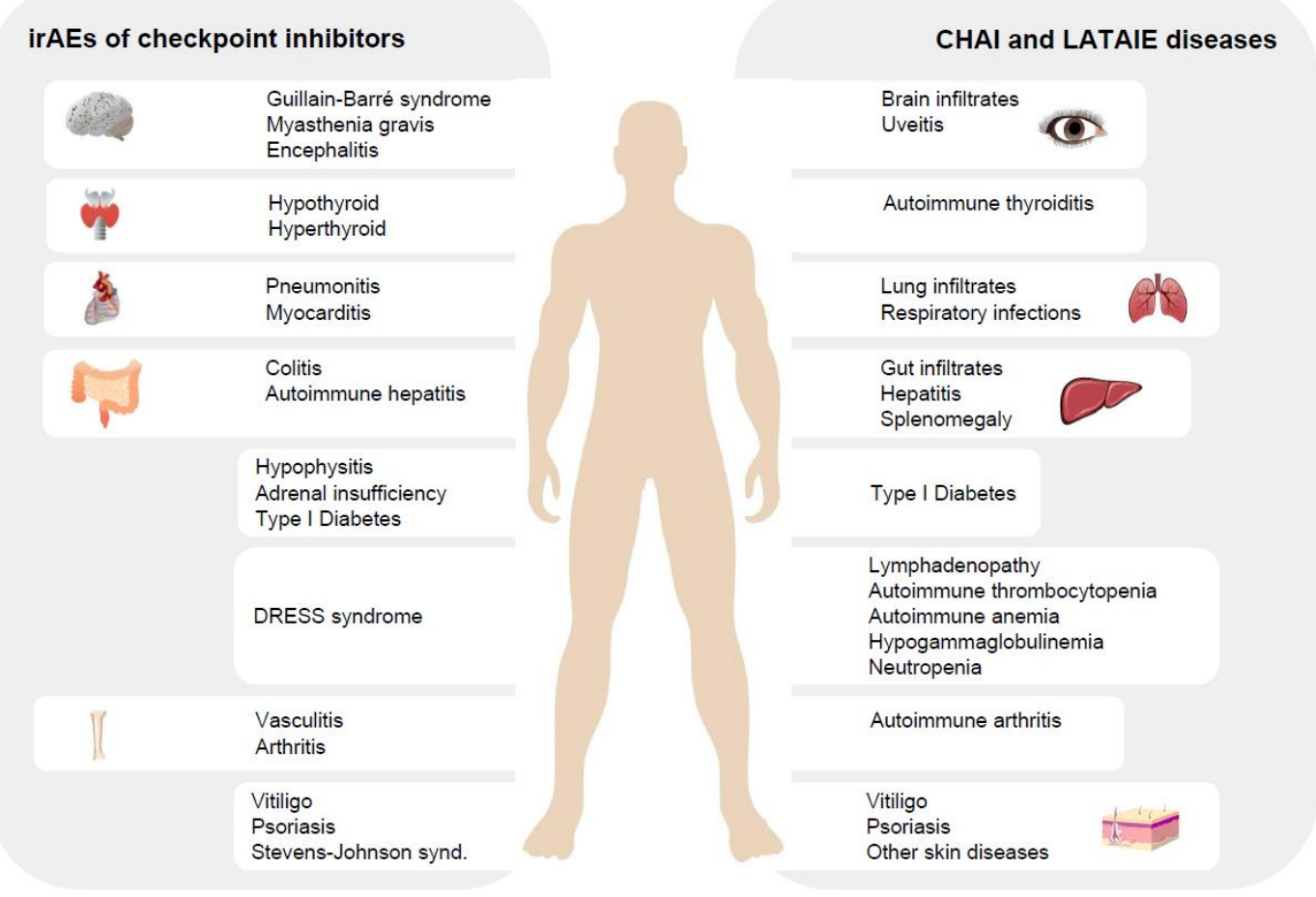


Thus, the theory that anti-CTLA-4 therapy has a similar mechanism to that occurring in inherited human CTLA4 haploinsufficiency [28] gains credibility by the overlapping clinical phenotypes of the genetic and iatrogenic CTLA-4 insufficiencies. Since the CTLA-4 receptor blockade cannot achieve long-lasting cancer regressions without breaking tolerance to healthy self-tissues, a therapeutic paradigm shift is required.

Consistent with this, June, Warshauer and Bluestone recently asked the question whether autoimmunity is the Achilles' heel of cancer immunotherapy [59]. They argued that the true incidence of autoimmune complications is probably underestimated following cancer immunotherapy because most cancer trials follow patients for only a brief time after enrollment and because patients who died from their cancer are not included. June et al. predicted that the incidence of immunotoxicity will likely continue to rise as these therapies become more widely used. Therefore, they warned that cancer immunotherapy is "a doubleedged sword in which patients and clinicians must weigh the risk of immunotoxicity against the benefit of tumor destruction".

Basic, simpler approaches could convert nonresponders into responders by increasing the therapeutic index of ICls

Fusi and Dalgleish emphasized recently that there must be a place for back to basic, simpler approaches, which might just prime the tumor environment to respond better to checkpoint inhibitors, increase the therapeutic index and convert nonresponders into responders [60]. In fact, spectacular therapeutic improvement in childhood cancer was achieved through "a reassessment of the tools in hand" applying them as combination therapies [61].

A combination therapy consisting of basic low tech approaches and immune checkpoint drugs in off-label low doses seems to be safer and more efficient in advanced cancer patients, than the currently used approved protocols. The idea for this protocol came from a groundbreaking but neglected proof-of-principle paper, which demonstrated augmented graft-versus-malignancy (GVM) effects that reversed the relapse of malignancy without worsening the graft-versus-host-disease (GVHD) by a CTLA-4 blockade [62].

Similarly, in the face of an ipilimumab induced pan-lymphocytic activation, a therapeutic paradigm shift was suggested. The task is not desperately trying to put the genie back in the bottle by immune suppressive treatments, but harnessing of the immense forces liberated by the anti-CTLA-4 antibody blockade for a GVM effect [24]. This resembles the use of 
allogeneic lymphocytes following allogeneic stem cell transplantation known as donor lyphocyte infusion. Slavin et al. suggested that a finely tuned, low-dose ipilimumab 0.3 $\mathrm{mg} / \mathrm{kg}$ ) therapy could induce a safe and effective auto-GVHD without compromising the long lasting objective to eradicate tumors throughout the body at the stage of minimal residual disease [27]. This way, the same goal could be achieved by an antibody-based drug as by adoptive transfer of alloreactive donor lymphocytes but without of course severe GVHD. Based on earlier observations [63] optimal graft-versus-leukemia (GVL) or GVM effects with no risk of hazardous GVHD could probably be accomplished by $0.3 \mathrm{mg} / \mathrm{kg}$ dose of ipilimumab.

Since then, a phase 3 trial compared a high-dose ipilimumab $(10 \mathrm{mg} / \mathrm{kg}$; 33.3 times higher dose than that of suggested by Slavin) to placebo in patients who had undergone complete resection of stage III melanoma [64]. In the ipilimumab group the 5-year recurrence-free survival rate was $41 \%$, but only $30 \%$ in the placebo group. In the ipilimumab group the 5 year overall survival rate was $65 \%$ compared to $54 \%$ in the placebo group. In the ipilimumab group $41.6 \%$ of the patients had irAEs of grade 3 or 4 , while only in $2.7 \%$ in the placebo group. Importantly, 5 patients (1.1\%) died due to irAEs in the ipilimumab group. Notwithstanding, the high-dose $(10 \mathrm{mg} / \mathrm{kg}$ ) adjuvant ipilimumab subsequently gained US Food and Drug Administration (FDA) approval.

\section{Proof-of-principle of low-dose ICI therapy demonstrated in a stage 4 triple negative breast cancer patient}

The low-dose ICl idea was adopted by Kleef et al. to stage IV cancer patients. The proof-ofprinciple was first demonstrated in a heavily pre-treated triple negative breast cancer (TNBC) patient with far advanced pulmonary metastases and severe shortness of breath, who had exhausted all conventional treatment [65] [66]. The patient was treated with a safe, off-label low-dose immune checkpoint blockade including ipilimumab $(0.3 \mathrm{mg} / \mathrm{kg})$ combined with nivolumab $(0.5 \mathrm{mg} / \mathrm{kg})$. This was complemented with moderate-dose interleukin-2 treatment under taurolidine protection and loco regional- and whole body hyperthermia without classical chemotherapy. The patient went into complete remission of her lung metastases and all cancer related symptoms vanished with transient WHO I-II diarrhea and skin rash. A total gene expression analysis of a metastatic axillary lymph node demonstrated that several checkpoint genes were over-expressed even after one year past initiation of 
therapy. The patient remained alive for 27 months after the start of treatment, with recurrence of metastases as a sternal mass, and up to $3 \mathrm{~cm}$ pleural metastases, which finally classified this patient having a mixed overall response.

Evidently, this TNBC patient with such far advanced lung metastasis would have had an extremely limited expected survival. Therefore, her response to the low-dose immune checkpoint blockade was exceptional.

"Rare cancer successes should instigate 'exceptional' research efforts" [67]. In many clinical trials there were rare patients whose advanced cancer vanished for many months or even for years. Previous NCl director and Nobel laureate Harold Varmus stated that we can really learn from such "exceptional responders" since they may explain why in certain patients a drug sometimes induces unexpected dramatic improvement, which then could be beneficial for more people.

Since the first treatment of the TNBC patient, a further 84 stage IV cancer patients with a variety of cancer types, have been treated safely with the off-label low-dose immune checkpoint blockade. The overall response (OR) rate of this patient group was $48.53 \%$ (including $17.19 \%$ of complete response (CR), 19.21\% partial response (PR) and $12.13 \%$ stabile disease (SD)) [68].

Recently, Sen et al. from the University of Texas M.D. Anderson Cancer Center confirmed the rationale for our low-dose immune checkpoint blockade protocol [69]. They demosntrated that despite a dose-dependent increase in irAEs no improvement in progression-free survival (PFS), overall survival (OS), or disease control rate (DCR) were identified with escalating doses of ICls. Sen at al. concluded that lower doses may reduce toxicity and cost without compromising disease control or survival. This is consistent with our results.

Sen et al., furthermore, have established a "prognostic scoring system" to help select patients [70]. The system is based on an analysis of 172 patients with advanced metastatic cancer who were enrolled in phase I clinical trials: 105 of them received anti-CTLA-4 drugs, while the rest received anti-PD-1 agents. The authors found 7 factors that predicted the best responding patients. One of us (RWM) rearranged these factors by their hazard ratios (HR) indicating the increased risk of death associated with each factor. In those who had zero, one or at most two risk factors, the median survival was over two years and about half of 
that group was still alive three years after receiving immune therapy. Actually, after twoyears of starting treatment none of these patients had died.

One can speculate that with better patient selection and lower doses of $\mathrm{ICl}$, this treatment could realistically fulfill the promise of Carl H. June, MD, the "father" of CAR T-cell therapy, that the present moment is only "the tip of the iceberg" of effective immunotherapy of cancer [59].

Importantly, our protocol consists only of approved drugs and treatments. Therefore, our prediction that low-dose ICl induced autoimmune T-cells are powerful therapeutic tools can be confirmed or refuted in prospective controlled clinical trials. (text words: 3912)

\section{Conclusions}

The risks of the ICls is a massively understudied area. Following the discovery of two new genetic diseases of CTLA-4 checkpoint insufficiency, the pan T cell activation theory of antiCTLA-4 therapy gains credibility. A therapeutic paradigm shift is required. Back to basic, simpler approaches (such as for example regional hyperthermia [71]) will increase the therapeutic index of $\mathrm{ICls}$ and convert nonresponders into responders. Harnessing of the immense forces liberated by the ICI blockade by an off-label low-dose ipilimumab and nivolumab therapy supplemented with IL-2 treatment and hyperthermia could induce a safe and effective GVM. The proof-of-principle of such an approach was demonstrated in several advanced metastatic cancer patients whose overall response (OR) rate was $48.53 \%$ without dangerous autoimmune side effects. Since the low-dose $\mathrm{ICI}$ protocol consists only of approved drugs and treatments our prediction that low-dose $\mathrm{ICl}$ induced autoimmune T-cells are powerful therapeutic tools can be confirmed or refuted in controlled clinical trials.

(words: 153)

\section{Declarations of interest:}

TB is a partner and CSO of PRET Therapeutics Ltd developing the patented low-dose ICI combination therapy. All other authors have none to declare.

\section{Acknowledgements}

For the critical questions and comments which were very helpful in the writing of the manuscript we would like to thank the late Professor Melvin Cohn, Ph.D., Salk Institute for Biological Studies 10010 N Torrey Pines Rd, La Jolla, CA 92037. 
This research did not receive any specific grant from funding agencies in the public, commercial, or not-for-profit sectors.

\section{References}

1. Thangavelu G, Gill RG, Boon L, Ellestad KK, Anderson CC. Control of in vivo collateral damage generated by T cell immunity. J Immunol. 2013;191(4):1686-91. Epub 2013/07/16. doi: 10.4049/jimmunol.1203240. PubMed PMID: 23851694.

2. Topalian SL. Targeting immune checkpoints in cancer therapy. JAMA. 2017. doi: 10.1001/jama.2017.14155.

3. Bakacs T, Kristof K, Mehrishi JN, Szabados T, Kerepesi C, Regoes E, et al. The Fallacy of Tumor Immunology http://arxiv.org/abs/1601.02404: Cornell University Library; 2016 [updated 2016].

4. Allison JP. Immune Checkpoint Blockade in Cancer Therapy: The 2015 Lasker-DeBakey Clinical Medical Research Award. JAMA. 2015:1113-5. doi: 2436310

[pii];10.1001/jama.2015.11929 [doi].

5. Postow MA, Callahan MK, Wolchok JD. Immune Checkpoint Blockade in Cancer Therapy. J Clin Oncol. 2015;33(17):1974-82. doi: JCO.2014.59.4358 [pii];10.1200/JCO.2014.59.4358 [doi].

6. Kelly PN. The Cancer Immunotherapy Revolution. Science. 2018;359(6382):1344-5. Epub 2018/03/24. doi: 10.1126/science.359.6382.1344. PubMed PMID: 29567702.

7. Peggs KS, Quezada SA, Allison JP. Cell intrinsic mechanisms of T-cell inhibition and application to cancer therapy. Immunol Rev. 2008;224:141-65. Epub 2008/09/02. doi: 10.1111/j.1600-065X.2008.00649.x. PubMed PMID: 18759925.

8. Fuertes Marraco SA, Neubert NJ, Verdeil G, Speiser DE. Inhibitory Receptors Beyond T Cell Exhaustion. Front Immunol. 2015;6:310. Epub 2015/07/15. doi:

10.3389/fimmu.2015.00310. PubMed PMID: 26167163; PubMed Central PMCID: PMCPMC4481276.

9. Schildberg FA, Klein SR, Freeman GJ, Sharpe AH. Coinhibitory Pathways in the B7CD28 Ligand-Receptor Family. Immunity. 2016;44(5):955-72. Epub 2016/05/19. doi: 10.1016/j.immuni.2016.05.002. PubMed PMID: 27192563; PubMed Central PMCID: PMCPMC4905708.

10. Sinclair N.R.S., Chan PL. Regulation of the Immune Response. IV. The Role of the FcFragment in Feedback Inhibition by Antibody. In, Morphological and Functional Aspects of Immunity. Advances in Experimental Medicine and Biology. 12. Boston, MA: Springer; 1971; p. 609-615.

11. Paraskevas F, Lee ST, Orr KB, Israels LG. A receptor for Fc on mouse B-lymphocytes. J Immunol. 1972;108(5):1319-27. Epub 1972/05/01. PubMed PMID: 4555113.

12. Amigorena S, Bonnerot C, Drake JR, Choquet D, Hunziker W, Guillet JG, et al. Cytoplasmic domain heterogeneity and functions of IgG Fc receptors in B lymphocytes. Science. 1992;256(5065):1808-12. Epub 1992/06/26. PubMed PMID: 1535455.

13. Klaus GG, Bijsterbosch MK, O'Garra A, Harnett MM, Rigley KP. Receptor signalling and crosstalk in B lymphocytes. Immunol Rev. 1987;99:19-38. Epub 1987/10/01. PubMed PMID: 2824341.

14. Sinclair NR, Anderson CC. Co-stimulation and co-inhibition: equal partners in regulation. Scand J Immunol. 1996;43(6):597-603. Epub 1996/06/01. PubMed PMID: 8658047. 
15. Sinclair NR, Panoskaltsis A. Antibody response and its regulation. Curr Opin Immunol. 1988;1(2):228-35. Epub 1988/12/01. PubMed PMID: 3077306.

16. Sinclair NR. How many signals are enough? Cell Immunol. 1990;130(1):204-12;

discussion 13-35. Epub 1990/10/01. PubMed PMID: 2204480.

17. Sinclair NR. Lymphocytic antigen receptors transmit positive signals! Scand J Immunol. 1990;31(6):665-71. Epub 1990/06/01. PubMed PMID: 2192436.

18. Cohn M. A Commentary on a Workshop 'To Reveal the Foundational Concepts of Immune Regulation' (i.e. Tolerance). Scand J Immunol. 2017;85(5):312-20. Epub 2017/02/16. doi: 10.1111/sji.12536. PubMed PMID: 28199746.

19. Anderson CC. Time, space and contextual models of the immunity tolerance decision: bridging the geographical divide of Zinkernagel and Hengartner's 'Credo 2004'. Scand J Immunol. 2006;63(4):249-56. Epub 2006/04/21. doi: 10.1111/j.1365-3083.2006.01742.x. PubMed PMID: 16623924.

20. Cohn M. On 'Credo 2004' as viewed under the 'development-context' model of Colin Anderson. Scand J Immunol. 2006;64(2):97-103. Epub 2006/07/27. doi: 10.1111/j.13653083.2006.01790.x. PubMed PMID: 16867154.

21. Blackburn SD, Shin H, Haining WN, Zou T, Workman CJ, Polley A, et al. Coregulation of CD8+ T cell exhaustion by multiple inhibitory receptors during chronic viral infection. Nat Immunol. 2009;10(1):29-37. Epub 2008/12/02. doi: 10.1038/ni.1679. PubMed PMID: 19043418; PubMed Central PMCID: PMCPMC2605166.

22. Hodi FS, O'Day SJ, McDermott DF, Weber RW, Sosman JA, Haanen JB, et al. Improved survival with ipilimumab in patients with metastatic melanoma. $\mathrm{N}$ Engl J Med. 2010;363(8):711-23. doi: NEJMoa1003466 [pii];10.1056/NEJMoa1003466 [doi].

23. Suntharalingam G, Perry MR, Ward S, Brett SJ, Castello-Cortes A, Brunner MD, et al. Cytokine storm in a phase 1 trial of the anti-CD28 monoclonal antibody TGN1412. N Engl J Med. 2006;355(10):1018-28.

24. Bakacs T, Mehrishi JN, Moss RW. Ipilimumab (Yervoy) and the TGN1412 catastrophe. Immunobiology. 2012;217(6):583-9. doi: S0171-2985(11)00136-7

[pii];10.1016/j.imbio.2011.07.005 [doi].

25. Szabados T, Bakacs T. Sufficient to recognize self to attack non-self: Blueprint for a one-signal T cell model. Journal of Biological Systems. 2011;19(2):299-317.

26. Bakacs T, Mehrishi JN, Szabo M, Moss RW. Interesting possibilities to improve the safety and efficacy of ipilimumab (Yervoy). Pharmacol Res. 2012;66(2):192-7. doi: S10436618(12)00064-3 [pii];10.1016/j.phrs.2012.03.015 [doi].

27. Slavin S, Moss RW, Bakacs T. Control of minimal residual cancer by low dose ipilimumab activating autologous anti-tumor immunity. Pharmacol Res. 2013. doi: S10436618(13)00172-2 [pii];10.1016/j.phrs.2013.10.004 [doi].

28. Bakacs T, Mehrishi JN. Anti-CTLA-4 therapy may have mechanisms similar to those occurring in inherited human CTLA4 haploinsufficiency. Immunobiology. 2014;220:624-5. doi: S0171-2985(14)00269-1 [pii];10.1016/j.imbio.2014.11.019 [doi].

29. Ellestad KK, Lin J, Boon L, Anderson CC. PD-1 Controls Tonic Signaling and Lymphopenia-Induced Proliferation of T Lymphocytes. Front Immunol. 2017;8:1289. Epub 2017/10/28. doi: 10.3389/fimmu.2017.01289. PubMed PMID: 29075267; PubMed Central PMCID: PMCPMC5643416.

30. Grossman Z, Paul WE. Adaptive cellular interactions in the immune system: the tunable activation threshold and the significance of subthreshold responses. Proc Natl Acad 
Sci U S A. 1992;89(21):10365-9. Epub 1992/11/01. PubMed PMID: 1438221; PubMed Central PMCID: PMCPMC50339.

31. Grossman Z, Paul WE. Dynamic tuning of lymphocytes: physiological basis, mechanisms, and function. Annu Rev Immunol. 2015;33:677-713. Epub 2015/02/11. doi: 10.1146/annurev-immunol-032712-100027. PubMed PMID: 25665077.

32. Ellestad KK, Anderson CC. Two Strikes and You're Out? The Pathogenic Interplay of Coinhibitor Deficiency and Lymphopenia-Induced Proliferation. J Immunol.

2017;198(7):2534-41. Epub 2017/03/23. doi: 10.4049/jimmunol.1601884. PubMed PMID: 28320914.

33. Ellestad KK, Thangavelu G, Haile Y, Lin J, Boon L, Anderson CC. Prior to Peripheral Tolerance, Newly Generated CD4 T Cells Maintain Dangerous Autoimmune Potential: Fasand Perforin-Independent Autoimmunity Controlled by Programmed Death-1. Front Immunol. 2018;9:12. Epub 2018/02/09. doi: 10.3389/fimmu.2018.00012. PubMed PMID: 29416537; PubMed Central PMCID: PMCPMC5787554.

34. Cohen IR. The cognitive paradigm and the immunological homunculus. Immunol Today. 1992;13(12):490-4. doi: 0167-5699(92)90024-2 [pii];10.1016/0167-5699(92)90024-2 [doi].

35. Madi A, Kenett DY, Bransburg-Zabary S, Merbl Y, Quintana FJ, Tauber Al, et al. Network theory analysis of antibody-antigen reactivity data: the immune trees at birth and adulthood. PLoS ONE. 2011;6(3):e17445. doi: 10.1371/journal.pone.0017445 [doi].

36. Cohen IR. Autoantibody repertoires, natural biomarkers, and system controllers. Trends Immunol. 2013;34(12):620-5. doi: S1471-4906(13)00080-X [pii];10.1016/j.it.2013.05.003 [doi].

37. Silverman GJ, Gronwall C, Vas J, Chen Y. Natural autoantibodies to apoptotic cell membranes regulate fundamental innate immune functions and suppress inflammation. Discov Med. 2009;8(42):151-6.

38. Couzin-Frankel J. Autoimmune diseases surface after cancer treatment. Science. 2017;358(6365):852. Epub 2017/11/18. doi: 10.1126/science.358.6365.852. PubMed PMID: 29146788.

39. Puzanov I, Diab A, Abdallah K, Bingham CO, Brogdon C, Dadu R, et al. Managing toxicities associated with immune checkpoint inhibitors: consensus recommendations from the Society for Immunotherapy of Cancer (SITC) Toxicity Management Working Group. Journal for ImmunoTherapy of Cancer. 2017;5(1):95. doi: 10.1186/s40425-017-0300-z. 40. Brahmer JR, Lacchetti C, Schneider BJ, Atkins MB, Brassil KJ, Caterino JM, et al. Management of Immune-Related Adverse Events in Patients Treated With Immune Checkpoint Inhibitor Therapy: American Society of Clinical Oncology Clinical Practice Guideline. J Clin Oncol. 2018:1714-68. Epub 2018/02/15. doi: 10.1200/JCO.2017.77.6385. PubMed PMID: 29442540.

41. Tawbi HA, Forsyth PA, Algazi A, Hamid O, Hodi FS, Moschos SJ, et al. Combined Nivolumab and Ipilimumab in Melanoma Metastatic to the Brain. N Engl J Med. 2018;379(8):722-30. Epub 2018/08/23. doi: 10.1056/NEJMoa1805453. PubMed PMID: 30134131.

42. Bertrand A, Kostine M, Barnetche $T$, Truchetet ME, Schaeverbeke T. Immune related adverse events associated with anti-CTLA-4 antibodies: systematic review and meta-analysis. BMC Med. 2015;13:211. doi: 10.1186/s12916-015-0455-8 [doi];10.1186/s12916-015-0455-8 [pii]. 
43. Widmann G, Nguyen VA, Plaickner J, Jaschke W. [Adverse effects of immunotherapy : Clinical aspects, radiological and nuclear medicine results]. Radiologe. 2017. Epub 2017/07/25. doi: 10.1007/s00117-017-0285-0. PubMed PMID: 28733704.

44. Khoja L, Day D, Wei-Wu Chen T, Siu LL, Hansen AR. Tumour- and class-specific patterns of immune-related adverse events of immune checkpoint inhibitors: a systematic review. Ann Oncol. 2017;28(10):2377-85. Epub 2017/09/26. doi: 10.1093/annonc/mdx286. PubMed PMID: 28945858.

45. Spain L, Larkin J. Combination immune checkpoint blockade with ipilimumab and nivolumab in the management of advanced melanoma. Expert Opin Biol Ther. 2016;16(3):389-96. Epub 2016/01/12. doi: 10.1517/14712598.2016.1141195. PubMed PMID: 26750801.

46. Kumar V, Chaudhary N, Garg M, Floudas CS, Soni P, Chandra AB. Current Diagnosis and Management of Immune Related Adverse Events (irAEs) Induced by Immune Checkpoint Inhibitor Therapy. Front Pharmacol. 2017;8:49. Epub 2017/02/24. doi:

10.3389/fphar.2017.00049. PubMed PMID: 28228726; PubMed Central PMCID: PMCPMC5296331.

47. Larkin J, Chiarion-Sileni V, Gonzalez R, Grob JJ, Cowey CL, Lao CD, et al. Combined Nivolumab and Ipilimumab or Monotherapy in Untreated Melanoma. N Engl J Med. 2015;373(1):23-34. doi: 10.1056/NEJMoa1504030. PubMed PMID: 26027431.

48. Teply BA, Lipson EJ. Identification and management of toxicities from immune checkpoint-blocking drugs. Oncology (Williston Park). 2014;28 Suppl 3:30-8. Epub 2014/11/12. PubMed PMID: 25384885.

49. Curran MA, Callahan MK, Subudhi SK, Allison JP. Response to "Ipilimumab (Yervoy) and the TGN1412 catastrophe". Immunobiology. 2012;217(6):590-2. doi:

10.1016/j.imbio.2011.11.001. PubMed PMID: 22459268.

50. Downey SG, Klapper JA, Smith FO, Yang JC, Sherry RM, Royal RE, et al. Prognostic factors related to clinical response in patients with metastatic melanoma treated by CTLassociated antigen-4 blockade. Clin Cancer Res. 2007;13(22 Pt 1):6681-8. doi: 10780432.CCR-07-0187 [pii];10.1158/1078-0432.CCR-07-0187 [doi].

51. Haratani $K$, Hayashi $H$, Chiba $Y$, et al. Association of immune-related adverse events with nivolumab efficacy in non-small-cell lung cancer. JAMA Oncology. 2018;4(3):374-8. doi: 10.1001/jamaoncol.2017.2925.

52. Beura LK, Hamilton SE, Bi K, Schenkel JM, Odumade OA, Casey KA, et al. Normalizing the environment recapitulates adult human immune traits in laboratory mice. Nature. 2016;532(7600):512-6. Epub 2016/04/21. doi: 10.1038/nature17655. PubMed PMID: $27096360 ;$ PubMed Central PMCID: PMCPMC4871315.

53. Freitas AA, Rocha B. Population biology of lymphocytes: the flight for survival. Annu Rev Immunol. 2000;18:83-111.

54. Bakacs T, Mehrishi JN, Szabados T, Varga L, Szabo M, Tusnady G. T cells survey the stability of the self: a testable hypothesis on the homeostatic role of TCR-MHC interactions. Int Arch Allergy Immunol. 2007;144(2):171-82. doi: 10.1159/000103282. PubMed PMID: 17541288.

55. Kuehn HS, Ouyang W, Lo B, Deenick EK, Niemela JE, Avery DT, et al. Immune dysregulation in human subjects with heterozygous germline mutations in CTLA4. Science. 2014;345(6204):1623-7. doi: science.1255904 [pii];10.1126/science.1255904 [doi]. 
56. Lo B, Abdel-Motal UM. Lessons from CTLA-4 deficiency and checkpoint inhibition. Curr Opin Immunol. 2017;49(Supplement C):14-9. doi: https://doi.org/10.1016/i.coi.2017.07.014.

57. Lo B, Fritz JM, Su HC, Uzel G, Jordan MB, Lenardo MJ. CHAl and LATAIE: new genetic diseases of CTLA-4 checkpoint insufficiency. Blood. 2016;128(8):1037-42. Epub 2016/07/16. doi: 10.1182/blood-2016-04-712612. PubMed PMID: 27418640; PubMed Central PMCID: PMCPMC5000841.

58. Voskens CJ, Goldinger SM, Loquai C, Robert C, Kaehler KC, Berking C, et al. The price of tumor control: an analysis of rare side effects of anti-CTLA-4 therapy in metastatic melanoma from the ipilimumab network. PLoS One. 2013;8(1):e53745. doi: 10.1371/journal.pone.0053745. PubMed PMID: 23341990; PubMed Central PMCID: PMCPMC3544906.

59. June $\mathrm{CH}$, Warshauer JT, Bluestone JA. Is autoimmunity the Achilles' heel of cancer immunotherapy? Nat Med. 2017;23(5):540-7. doi: 10.1038/nm.4321. PubMed PMID: 28475571.

60. Fusi A, Dalgleish A. The importance for immunoregulation for long-term cancer control. Future Oncol. 2017;13(18):1619-32. Epub 2017/08/05. doi: 10.2217/fon-2017-0085. PubMed PMID: 28776411.

61. Ledford H. Cocktails for cancer with a measure of immunotherapy. Nature. 2016;532(7598):162-4. doi: 532162a [pii];10.1038/532162a [doi].

62. Bashey A, Medina B, Corringham S, Pasek M, Carrier E, Vrooman L, et al. CTLA4 blockade with ipilimumab to treat relapse of malignancy after allogeneic hematopoietic cell transplantation. Blood. 2009;113(7):1581-8. doi: blood-2008-07-168468 [pii];10.1182/blood2008-07-168468 [doi].

63. Wolchok JD, Neyns B, Linette G, Negrier S, Lutzky J, Thomas L, et al. Ipilimumab monotherapy in patients with pretreated advanced melanoma: a randomised, double-blind, multicentre, phase 2, dose-ranging study. Lancet Oncol. 2010;11(2):155-64. doi: S14702045(09)70334-1 [pii];10.1016/S1470-2045(09)70334-1 [doi].

64. Eggermont AM, Chiarion-Sileni V, Grob JJ, Dummer R, Wolchok JD, Schmidt H, et al. Prolonged Survival in Stage III Melanoma with Ipilimumab Adjuvant Therapy. N Engl J Med. 2016;375(19):1845-55. doi: 10.1056/NEJMoa1611299. PubMed PMID: 27717298.

65. Kleef R, Moss RW, Szasz AM, Bohdjalian A, Bojar H, Bakacs T. From Partial to Nearly Complete Remissions in Stage IV Cancer Administering Off-label Low-Dose Immune Checkpoint Blockade in Combination with High Dose Interleukin-2 and Fever Range Whole Body Hyperthermia. ASCO; Chicago, USA: J. Clin. Oncol. 34, suppl; abstr e23111; 2016.

66. Kleef R, Moss R, Szasz AM, Bohdjalian A, Bojar H, Bakacs T. Complete Clinical Remission of Stage IV Triple-Negative Breast Cancer Lung Metastasis Administering LowDose Immune Checkpoint Blockade in Combination With Hyperthermia and Interleukin-2. Integr Cancer Ther. 2018:1534735418794867. Epub 2018/09/09. doi: 10.1177/1534735418794867. PubMed PMID: 30193538.

67. Kaiser J. Biomedicine. Rare cancer successes spawn 'exceptional' research efforts. Science. 2013;340(6130):263. doi: 340/6130/263 [pii];10.1126/science.340.6130.263 [doi]. 68. Kleef R. Exogenous and endogenous hyperthermia combining low-dose checkpointinhibitors with interleukin-2 (IL-2) and fever range whole body and local regional hyperthermia in stage IV cancer. Oncothermia Journal 2018;23.

69. Sen S, Hess KR, Hong DS, Naing A, Huang L, Meric-Bernstam F, et al. Impact of immune checkpoint inhibitor dose on toxicity, response rate, and survival: A pooled analysis 
of dose escalation phase 1 trials. J Clin Oncol. 2018;36(15_suppl):3077-. doi:

10.1200/JCO.2018.36.15_suppl.3077.

70. Sen S, Hess K, Hong DS, Naing A, Piha-Paul S, Janku F, et al. Development of a prognostic scoring system for patients with advanced cancer enrolled in immune checkpoint inhibitor phase 1 clinical trials. Br J Cancer. 2018;118:763. doi: 10.1038/bjc.2017.480 https://www.nature.com/articles/bjc2017480\#supplementary-information.

71. Issels RD, Lindner LH, Verweij J, Wessalowski R, Reichardt P, Wust P, et al. Effect of Neoadjuvant Chemotherapy Plus Regional Hyperthermia on Long-term Outcomes Among Patients With Localized High-Risk Soft Tissue Sarcoma: The EORTC 62961-ESHO 95 Randomized Clinical Trial. JAMA Oncol. 2018. Epub 2018/02/17. doi:

10.1001/jamaoncol.2017.4996. PubMed PMID: 29450452. 